\title{
Effects of learning contexts on implicit and explicit learning
}

\author{
YUH-SHIOW LEE \\ The Chinese University of Hong Kong, Shatin, Hong Kong
}

\begin{abstract}
Two parallel tasks involving rule learning were identified in Experiment $1 \mathrm{~A}$ and were used to assess implicit and explicit learning. In both tasks, subjects had to input numbers in order to reach the target values of outputs. The relationship between inputs and outputs was either simple (in the simple task) or complex (in the complex task), and the way in which target values were presented could be in the form of either numbers (in the simple task) or lines (in the complex task). Experiment 1B examined the validity of the explicit measure in the complex task. Experiments $2-4$ investigated the interaction between learning contexts and the simple/complex learning tasks. Verbalization and instructions to search for the rules facilitated the simple-task learning and hurt or have no effect on the complex-task learning. In the observational-learning condition, no learning occurred for the simple task, and the complex task learning was impaired. These results suggest that the complex task and simple task involve two distinct learning systems. Other implications are also discussed.
\end{abstract}

We are probably all familiar with the distinction between two kinds of thinking and learning processes. Some learning and decisions, in which we are fully aware of our thinking processes, seem rational and logical. We can explain these to others either on the basis of our judgment or according to what we have learned. Other learning seems to be based on guessing, feeling, or intuition. We learn and make decisions without knowing exactly what we have learned or how we have reached these decisions. This is parallel to the distinction between explicit learning and implicit learning recently proposed by some investigators (e.g., Berry \& Broadbent, 1987, 1988; Mathews et al., 1989; Reber, 1989a; Schacter, 1987). The occurrence of explicit (or implicit) learning is evidenced by showing that subjects' behavior changes with (or without) corresponding explicit knowledge, and the explicit knowledge is measured by the degree to which subjects can express what they have learned (e.g., Berry \& Broadbent, 1984; Lewicki, 1986b).

Implicit-learning paradigms include learning of a complex rule (Reber, 1967, 1976; Reber \& Allen, 1978; Reber \& Lewis, 1977), probability learning (Millward \& Reber, 1972; Reber \& Millward, 1971), covariation learning (Lewicki, 1982, 1986b; Lewicki, Czyzewska, \&

This research was based on part of my doctoral dissertation submitted to the State University of New York at Stony Brook. Experiment 4 was conducted while I was an associate professor at Soochow University, Taipei, Taiwan. I wish to thank the reviewers Richard Carlson, Michael Masson, and Scott Allen for their helpful comments on previous versions of this article. I extend special thanks to my advisor, Robert Frick, to the dissertation committee, Edward Casey, Nancy Franklin, and Nancy Squires, and also to Douglas Vakoch, for their extensive comments on earlier versions of this article. Correspondence should be addressed to Y.-S. Lee, Department of Psychology, The Chinese University of Hong Kong, Shatin, New Territories, Hong Kong (e-mail: yslee@cuhk.hk).
Hoffman, 1987), and learning of complex rule systems (Berry \& Broadbent, 1984; Broadbent, 1977; Broadbent \& Aston, 1978; Broadbent, FitzGerald, \& Broadbent, 1986). The studies listed above share the same approach: they show implicit learning by demonstrating a dissociation between subjects' performance on a task and their corresponding explicit knowledge. This approach has generated a major controversy in implicit-learning research. In particular, there are problems with the measurement of explicit knowledge in these studies. Thus, the definitions and measurement of explicit knowledge are central issues and are the focus of most of the critiques of implicitlearning research (Brody, 1989; Dulany, Carlson, \& Dewey, 1984, 1985; Mathews, 1990; Perruchet \& Amorim, 1992; Perruchet \& Pacteau, 1990; Reber, 1989b; Reber, Allen, \& Regan, 1985).

Two procedures, recognition and verbalization, have been used to measure explicit knowledge, but there are difficulties with both of them. Only a few studies adopted recognition or recognition-like procedures to measure explicit knowledge. Perruchet and Pacteau (1990) presented the main pieces of letter strings, which were thought to be the basis for judgments of grammaticality, as a target in the recognition-like task, and concluded that subjects were aware of the information that accounted for their grammaticality judgments. In other studies, subjects were instructed to mark the specific features of strings responsible for their decision concerning grammatical status (Carlson \& Dulany, 1985; Dulany et al., 1984). Once again, it was found that subjects' accuracy could be accounted for by this conscious measure.

Reber et al. (1985) and Mathews (1990), however, have pointed out the inappropriateness of recognition procedures as a means with which to measure explicit knowledge. In this kind of procedure, subjects may actually be able to make correct responses without accessing ex- 
plicit knowledge. For example, subjects can recognize or indicate the parts of letter strings that do not follow the rules without explicit knowledge of these rules. Therefore, this knowledge could still be implicit. Recognition does not necessarily measure explicit knowledge.

Almost all studies on implicit learning adopt verbal report as the measure of conscious information available to subjects. Three procedures have been used: interviews or questionnaires (Berry \& Broadbent, 1984, 1987, 1988; Broadbent, 1977; Broadbent \& Aston, 1978; Broadbent et al., 1986; Lewicki, 1986a, 1986b; Mathews, Buss, Chinn, \& Stanley, 1988; Mathews et al., 1989; Sanderson, 1989; Stanley, Mathews, Buss, \& KotlerCope, 1989); protocol analysis (Allen \& Reber, 1980; Reber \& Allen, 1978; Reber \& Lewis, 1977); and teachaloud, or giving subjects' recorded reports to yoked subjects (Mathews et al., 1988; Mathews et al., 1989; Stanley et al., 1989).

For the purpose of demonstrating implicit learning, verbal report seems a more appropriate procedure with which to measure explicit knowledge than recognition, and actually it has been used in most research. Still, researchers should be cautious about their measurement of subjects' verbalizable knowledge before concluding too quickly that some knowledge is inaccessible, since there is a major problem with the verbalization criterion as a measure of explicit knowledge. The difficulty lies in the fact that several reasons other than implicit learning could explain why subjects are unable to verbalize their knowledge about what they have learned. First, the subjects may forget to mention some of the information that guided their performance of the task. The problem of forgetting happens easily in postexperimental interviews and protocol-analysis procedures. Second, subjects may not be sufficiently motivated and articulate to communicate their knowledge about what they have learned (Lewicki, Hill, \& Bizot, 1988).

In sum, there are problems with the use of both the recognition procedure and the verbalization procedure for measuring explicit knowledge. The problem with the recognition procedure is that recognition can occur without awareness. Thus, using the recognition procedure to show that subjects are able to recognize the knowledge they have used seems an inappropriate way in which to demonstrate that implicit learning does not exist. The problem with the verbalization procedure is that other factors could account for the fact that subjects sometimes cannot verbalize their knowledge. To claim that there is implicit learning, one should be able to rule out these alternative explanations.

The first purpose of the present research was to yield a better methodological control for demonstrating the implicit-explicit distinction in learning. In particular, the present study provided more comparable measures of performance and explicit knowledge. Both measures assessed the same aspect of a task. Experiment 1A was designed to compare these two measures under various conditions in order to find both the factors that deter- mine the types of learning and two different tasks that involve primarily implicit learning and explicit learning. Experiment $1 \mathrm{~B}$ aimed at examining the validity of the explicit measure.

The second purpose was to examine how manipulations of learning contexts interact with the implicit learning and explicit learning. Also, the implicit-explicit learning distinction was tested further, and factors that dissociate or associate implicit and explicit learning were identified. By identifying these factors, one may also gain insight into how to improve performance on implicit and explicit learning. Experiments 2 and 3 investigated the effects of verbalization and instructions on searching for the rule. Experiment 4 looked at the effects of observational learning.

\section{AN OVERVIEW}

To measure subjects' explicit knowledge, the present study used a multiple-choice questionnaire asking subjects about simple quantitative relationships among variables. The subjects had to make a choice among all the possible relations among variables. They cannot make such choices on the basis of their implicit knowledge, because by definition, implicit knowledge lies outside subjects' awareness. In other words, subjects' knowledge measured by the multiple-choice questionnaire procedure is explicit knowledge. This relatively open-ended procedure reduces not only the problem of suggesting answers to subjects, as yes-no recognition procedures do, but also the problem that subjects may not have sufficient verbal skill to articulate their explicit knowledge. The problem of forgetting is also reduced by asking subjects only about simple relationships among variables.

Subjects' implicit knowledge was measured by the difference between their performance test on a task and their explicit knowledge about the same aspect of the task. If subjects have implicit knowledge about the task--in other words, if implicit learning occurs-the measure of their task performance should be greater than the measure of their explicit knowledge, provided that these two measurements measure exactly the same aspect of the stimulus structure. If only explicit learning occurs, the measures of their task performance should be either equal to the measures of the explicit knowledge (because subjects are fully aware of what they have learned) or worse than the measure of their explicit knowledge (because subjects may not use their explicit knowledge completely and efficiently).

To establish a compatible measurement of implicit and explicit knowledge in a given task, the present study abstracted the part of the information from subjects' task performance that was exactly the same as that from the multiple-choice questionnaire. By comparing these two measures, it would then be possible to identify a task that more likely involves primarily implicit learning, and another task that more likely involves primarily explicit learning. 


\section{The Tasks}

For all the tasks in the present study, the subjects were asked on each trial to input two numbers in order to reach certain values of two outputs. The equations relating the input numbers and output numbers were as follows:

Complex rules:

Output $1=(3.5 \times$ Input 1$)+(4 \times$ Input 2$)$

Output $2=(7.5 \times$ Input 2$)-(0.7 \times$ Input 1$)$.

Simple rules:

Output $1=0.5 \times$ Input 2

Output $2=3 \times$ Input 1 .

Subjects were allowed to choose two whole numbers from 1 to 10 for both Input 1 and Input 2. They were given feedback in two outputs, the values of which were determined by the two input numbers. The outputs given to subjects were in the form of either (1) lines with the length corresponding to the values of outputs or (2) numbers with corresponding values. For example, according to the first complex rule, if subjects input 10 and 5 for Input 1 and Input 2, in the line condition, two lines 55 and 45 units long (Output 1 and Output 2, respectively) were presented to them. In the number condition, two numbers 55 and 45 were presented. Without knowing the equations, the subjects tried to learn how to assign the input numbers by relating their input numbers to the feedback, in order to reach the target length or values.

In the learning phase, all subjects had to try to solve a total of 12 questions (i.e., reach 12 pairs of target outputs), and for each question, they had 30 chances. All 12 questions were based on a single rule, either complex or simple. Immediately after the learning phase, the subjects' performance was tested by 9 more questions in the same manner as in the learning phase, but without feedback. In the testing phase, the subjects had only one chance to input numbers for each question.

\section{The Measures of Explicit Knowledge}

The subjects' explicit knowledge was assessed by multiple-choice questionnaires. Eight questions tested the subjects' knowledge of the quantitative relationships between input values and output values. These 8 questions have the following form: If you want to increase (decrease) Output 1 (Output 2), then what will you do with Input 1 (Input 2)? The subjects had to choose from one of the following three answers: (1) always increase; (2) always decrease; and (3) stay the same (or I cannot do anything about it).

\section{The Measures of Performance Test}

In order to draw the information from the subjects' inputs of numbers that corresponds to that from the multiple-choice questions, the questions in the performance test were specially designed. There were nine questions in the performance test, five of which provided eight comparisons about Input 1 and Input 2 (the other four questions were fillers). These eight compar- isons compared input numbers between Questions 1 and 2, Questions 1 and 3, Questions 1 and 4, and Questions 1 and 5 for both Input 1 and Input 2 (see Table 1). These eight comparisons, which were based on the directional relations between any two numbers, matched the eight questions in the questionnaire. For example, if subjects' Input 1 for the pair 42 (Output 1), 30 (Output 2) was 3, and for the pair 55 (Output 1), 30 (Output 2) was 6 , then by comparing the two Input 1 numbers ( 3 and 6 ), one could decide what change subjects made on Input 1 when only Output 1 was increased (from 42 to 55), with no change in Output 2 . This information corresponded to the question "If you want to increase Output 1, then what will you do with Input l?"

\section{The Comparisons of Two Measures}

By comparing subjects' inputs for the different pairs of outputs, one could decide whether subjects' responses on the performance test followed the equations used to relate inputs and outputs. Subjects' responses about the same relationships on the questionnaire could also be assessed by using the same equations. Accordingly, correct percentages (the numbers of correct responses divided by the total of eight comparisons/questions) could be calculated for both the performance test and the questionnaire. Because the subjects gave answers in different ways for the performance test and the questionnaire, these two measures had different chance levels. For the questionnaire, there are three possible answers; thus, the chance level of giving the correct answer is .33. For the performance test, the subjects have a total of 10 numbers from which to choose, and the chance that they will choose the same numbers for both inputs is therefore. 1 . The chance that subjects will choose different numbers is .9 , with a .45 chance that the first number chosen will be larger than the second, and a .45 chance that the first number chosen will be smaller than the second. For all the reported results in the present study, the correct percentages were corrected for chance success before any analyses were carried out. The formula used to correct for chance success is: $P_{\text {corrected }}=\left(P_{\text {observed }}-P_{\text {chance }}\right) /$ $\left(1-P_{\text {chance }}\right)$, where $P$ is the correct percentage.

Table 1

An Example of the Eight Comparisons for Measuring Subjects" Performance on the Task

\begin{tabular}{crrrr}
\hline & \multicolumn{2}{c}{ Output } & \multicolumn{2}{c}{ Input } \\
\cline { 2 - 5 } Question & 1 & 2 & 1 & 2 \\
\hline 1 & 42 & 30 & $\rightarrow 3$ & $\rightarrow 6$ \\
2 & 55 & 30 & $\mathrm{Cl} \rightarrow 6$ & $\mathrm{C} 5 \rightarrow 7$ \\
3 & 16 & 30 & $\mathrm{C} 2 \rightarrow 4$ & $\mathrm{C} 6 \rightarrow 5$ \\
4 & 42 & 75 & $\mathrm{C} 3 \rightarrow 5$ & $\mathrm{C} 7 \rightarrow 8$ \\
5 & 42 & 6 & $\mathrm{C} 4 \rightarrow 9$ & $\mathrm{C} 8 \rightarrow 3$ \\
6 & 54 & 72 & 5 & 2 \\
7 & 18 & 9 & 7 & 7 \\
8 & 60 & 21 & 3 & 6 \\
9 & 20 & 36 & 1 & 6 \\
\hline
\end{tabular}

Note $-\mathrm{Cl}-\mathrm{C} 8=$ Comparison 1 to Comparison 8 . For example, $\mathrm{Cl}$ is the comparison between 3 and $6 ; \mathrm{C} 2$ is the comparison between 3 and 4 . 


\section{EXPERIMENT 1A}

Experiment $1 \mathrm{~A}$ was designed to examine under what conditions implicit and explicit learning occur. One of the most important characteristics of implicit learning is that the rule systems that subjects learn are complex and difficult to learn consciously or strategically (see Reber, 1989a). It also has been suggested that perceptual information is primarily responsible for implicit learning (Stadler, 1989), and that implicit learning is tied to surface characteristics (Berry \& Broadbent, 1988; Squire \& Frambach, 1990). From these findings, the complexity of rule systems and the nonsymbolic nature of perceptual discrimination seem to be two key characteristics of implicit learning. In contrast, explicit learning would be more likely to occur when the rule to be learned is simple and the task encourages symbolic processing.

For the tasks designed in the present study, rules used to determine the relationships between inputs and outputs could be either complex or simple, and the form of outputs could be either lines or numbers. Thus, there was a total of four possible combination conditions under which subjects could be tested. It was predicted that implicit learning was more likely to occur under the complex-rule-and-line condition, and that explicit learning was more easy to obtain from the simple-rule-andnumber condition.

\section{Method}

Subjects. In Experiments 1A, 2, and 3, the subjects were students from introductory psychology courses at SUNY at Stony Brook, participating voluntarily to fulfill part of their course requirements. Eighty-eight students participated in Experiment $1 \mathrm{~A}$.

Design. This experiment was a $2 \times 2 \times 2$ mixed design. The testing type (performance test vs. questionnaire) was a withinsubjects factor. The complexity of the rules used to determine the relationships between inputs and outputs (complex or simple) and the form of the outputs (lines or numbers) were two betweensubjects factors. Thus, there was a total of four possible combinations: complex rule-line, complex rule-number, simple rule-line, and simple rule-number. Twenty-two subjects were assigned to each one of the four conditions and were measured by both performance test and questionnaire.

Procedure. All subjects were run on an IBM personal computer. The subjects were given instructions on the computer screen. When the experiment started, the experimenter also explained to them the goals of the task. The subjects were not told anything about the patterns or equations. They were asked only to find the correct inputs for each pair of outputs.

During the learning phase, for each question, the target values were always available at the top of the screen. The subjects were asked to enter two numbers, one for Input 1 and the other for Input 2, and then the computer presented two output values, which were calculated from the subjects' input values. If the output values were different from the target values, the subjects were asked to try again. When a subject either reached the maximum of 30 trials or found the correct input values, the computer moved to the next question. The subjects were not told the correct answers when they failed to find them within 30 trials.

After subjects finished a total of 12 questions, they were told that they would be tested on what they had learned from the learning phase. They were then tested on 9 more questions in a similar manner without feedback, with only one chance per trial to input the two numbers. Following the performance test, they were given the multiple-choice questionnaires. They were again asked to respond on the basis of what they had learned during the learning phase.

\section{Results and Discussion}

The results are presented in Table 2, which shows both mean correct percentages and mean trials to targets in each condition. A three-way analysis of variance (ANOVA) was carried out on the correct percentages, with rule complexity (complex vs. simple), output form (line vs. number), and testing type (performance test vs. questionnaire) as factors. The effect of output form was significant $\left[F(1,84)=10.03, M S_{\mathrm{e}}=.23, p<.01\right]$. More importantly, both the two-way interaction between the form of output and the testing type and the three-way interaction were significant $\left[F(1,84)=6.21, M S_{\mathrm{e}}=\right.$ $.07, p<.05$ and $F(1,84)=19.44, M S_{\mathrm{e}}=.07, p<.001$, respectively].

Further analyses showed that for both the complex rule-line and simple rule-number conditions, the subjects' correct percentages on both questionnaire and performance test were better than chance $[t(21)=6.07$ and $t(21)=18.20$ for the complex rule-line condition, and $t(21)=3.86$ and $t(21)=3.80$ for the simple rulenumber condition, all $p \mathrm{~s}<.001 \mathrm{]}$. As to both the complex rule number and the simple rule-line conditions, except for the performance test on the simple rule-line condition $[t(21)=2.10, p<.05]$, all measures were at the chance level. These results indicate that almost no learning occurred in the complex rule-number and simple rule-line conditions. Thus, no further analysis was carried out for these two conditions. This is consistent with the finding that there were significantly fewer trials to target in both the complex rule-line and simple rulenumber conditions than there were in the complex rulenumber and simple rule line conditions (all $p s<.01$ ).

To find the conditions under which implicit and explicit learning occurred, the critical comparisons for this experiment were the differences between correct percentages for the performance test and questionnaire for each of the four conditions. A post hoc test (NewmanKeuls) revealed that in the complex rule line condition, the subjects did better on performance than on the questionnaire (at $p<.01$ ), indicating that implicit learning occurred. In the simple rule-number condition, no difference was found between performance test and questionnaire, indicating that the subjects' explicit knowledge could account for their performance test (i.e., that explicit learning occurred). Further, the Pearson correlation between the performance test and questionnaire

Table 2

Results for Experiment 1A: Mean Correct Percentages on the Performance Test $(P)$ and the Questionnaire $(Q)$, and Mean Trials to Targets $(T ; N=88)$

\begin{tabular}{lllr}
\hline \multicolumn{1}{c}{ Condition } & $P$ & $Q$ & $T$ \\
\hline Simple rule-number & .32 & .45 & 7.97 \\
Simple rule-line & .28 & .20 & 12.95 \\
Complex rule-number & .08 & .24 & 15.63 \\
Complex rule-line & .60 & .26 & 7.16 \\
\hline
\end{tabular}


was highly significant in the simple rule-number condition $(r=.74, p<.001)$, but was not significant in the complex rule-line condition. Accordingly, two procedurally parallel tasks involving primarily implicit learning (the complex rule-line condition) and explicit learning (the simple rule-number condition) were identified. These results were also consistent with the prediction that implicit learning happened in the condition in which the rules were complex and the task involved nonsymbolic perceptual discrimination, while explicit learning occurred when the rules were simple and the task involved symbolic processing.

For convenience, in all the following experiments, the complex rule-line condition task was labeled the complex task and the simple rule-number condition task was labeled the simple task. In addition, these two tasks were also used to examine the effects of different learning contexts on implicit and explicit learning.

\section{EXPERIMENT 1B}

Although the performance test and questionnaire assessed the same aspect of a task in this study, one methodological problem concerning the knowledge assessed by the questionnaire in the complex task needs to be further examined: Was it possible that the difference between the performance test and the questionnaire in the complex task was due to a lack of sensitivity in the assessment of explicit knowledge? In other words, did the questionnaire reveal all the explicit knowledge that affected performance?

The purpose of Experiment 1B was to verify the validity of the questionnaire in the complex task. If the questionnaire is a valid measure of explicit knowledge used in the performance test, subjects' scores on the questionnaire should not be worse than their scores on the performance test, when they are given the equations. In addition, those subjects who are told the equations should perform differently from the control subjects on at least one of the measures.

\section{Method}

Subjects and Design. Thirty-four students at the Chinese University of Hong Kong participated in this experiment. They were paid for their participation. This experiment was a $2 \times 2$ mixed design, with testing type (performance test vs. questionnaire) as the within-subjects factor. Subjects were randomly assigned to either the explicit-rule condition or the standard condition

Procedure. The procedure for the standard group in the present experiment was the same as that used in the complex rule-line condition of Experiment $1 \mathrm{~A}$. For the explicit-rule condition, the subjects were also run on an IBM personal computer and tested in the complex rule-line condition. They were given the following formula written on a piece of paper, and it was available to them throughout the whole time of the experiment:

$$
\begin{aligned}
& \text { Output } 1=(3.5 \times \text { Input } 1)+(4 \times \text { Input } 2) \\
& \text { Output } 2=(7.5 \times \text { Input } 2)-(0.7 \times \text { Input } 1) .
\end{aligned}
$$

The subjects were asked to complete the performance test and questionnaire according to the formula. To allow them to become famiiar with the nature of the formula, they were given two practice ques-
Table 3

Results for Experiment 1B: Mean Correct Percentages on the Performance Test $(P)$ and the Questionnaire $(Q ; N=34)$

\begin{tabular}{lcc}
\hline \multicolumn{1}{c}{ Group } & $P$ & $Q$ \\
\hline Standard & .69 & .37 \\
Explicit rule & .55 & .82
\end{tabular}

tions before the test. These two practice questions were given in the same way as those in the learning phase for the standard group.

\section{Results and Discussion}

A $2 \times 2$ ANOVA showed a main effect of learning condition [standard condition vs. explicit-rule condition; $\left.F(1,32)=4.42, M S_{\mathrm{e}}=.41, p<.05\right]$ and an interaction effect between learning condition and testing type [performance test vs. questionnaire; $F(1,32)=44.49$, $M S_{\mathrm{e}}=1.48, p<.001 ;$ see Table 3].

A post hoc test (Newman-Keuls) revealed that the standard group did significantly better on the performance test than on the questionnaire $(p<.001)$, indicating that implicit learning occurred. In the explicit-rule condition, on the other hand, subjects did significantly better on the questionnaire than on the performance test $(p<.001)$. There was also a significant difference between the standard group and the explicit-rule group on both the questionnaire $(p<.001)$ and the performance test $(p<.05)$.

It was not surprising that subjects in the explicit-rule group, who knew the equations, did better than those in the standard group on the questionnaire, or that the standard group replicated the findings of Experiment $1 \mathrm{~A}$. More important was the finding that for the explicit-rule group, the questionnaire was not only sensitive enough to assess the knowledge that affects the performance test, it was also more sensitive than the performance test. When they were explicitly told the exact relationship between inputs and outputs, subjects could do quite well on the questionnaire. However, this knowledge was more difficult to use in the performance test. In contrast to the simple task, the outputs in the complex task were presented in the form of lines. This could have caused subjects more difficulty in making use of the equations when doing the performance test. Moreover, the fact that the standard group did better than the explicit-rule group on the performance test suggests that experience with the task is more important for the performance test, whereas knowing the equations is not so crucial.

\section{EXPERIMENT 2}

In Experiment 2, we examined the effect of instructions to search for rules on both implicit and explicit learning. To my knowledge, prior to the present study, the effect of instructions has only been investigated on implicit learning (Reber, 1976). Without cont rasting this with the effect of instructions on explicit learning, it is difficult to evaluate how instructions work. Instructing subjects to search for a pattern may induce stress and anxiety, which may impair their performance in general. 
In this case, subjects will do worse on both the complex and the simple tasks. Another possibility is that instructions induce motivation and encourage subjects to use a conscious strategy, which leads them to do better on the simple task and impairs or has no effect on the complextask learning. More importantly, examining the effect of instructions on both the complex and simple tasks will provide an additional test of the proposal that these two tasks involve two separate learning systems.

\section{Method}

Subjects and Design. See Experiment $1 \mathrm{~A}$ for details regarding subjects. Experiment 2 was a $2 \times 2 \times 2$ mixed design. The task type (complex task vs. simple task) and instruction type (rule-finding instructions vs. standard instructions) were manipulated between subjects. The testing type (performance test vs. questionnaire) was a within-subjects factor. Eighty subjects were randomly assigned to one of the following four groups: the complex task with rulefinding instructions, the simple task with rule-finding instructions, and one group of control subjects for each task with standard instructions. There were 20 subjects in each group.

Procedure. Subjects in the complex task were tested in the same manner as those in the complex rule-line condition of Experiment $1 \mathrm{~A}$, and those in the simple task were tested in the same manner as those in the simple rule-number condition of Experiment 1A. Following the same procedures as those used in Experiment $1 \mathrm{~A}$, the standard-instruction subjects were not told anything about the patterns or equations. They were asked only to find the correct inputs for each pair of outputs. The subjects given the rulefinding instructions received the following extra instructions at the beginning of the experiment: "You also have to search for the patterns (rules), which determined the relationship of input values and the output values. You will be asked to report these patterns at the end of this experiment."

\section{Results and Discussion}

Both mean correct percentages and mean trials to targets are presented in Table 4 . There was no significant difference on the mean trials to targets among different conditions. A $2 \times 2 \times 2$ ANOVA was carried out on the correct percentages. There was a significant difference in the correct percentages between the performance test and the questionnaire $\left[F(1,76)=15.67, M S_{\mathrm{e}}=.06, p<\right.$ $.001]$. More importantly, there were interactions between task type (complex task vs. simple task) and instruction type [standard vs. rule-finding instructions; $\left.F(1,76)=6.62, M S_{\mathrm{e}}=.25, p<.05\right]$, and between task type and testing type [performance test vs. questionnaire; $\left.F(1,76)=39.39, M S_{\mathrm{e}}=.06, p<.001\right]$. No threeway interaction was found.

Planned comparisons showed that for the complex task, subjects in both the rule-finding instruction and the standard instruction groups did significantly better on the performance test than on the questionnaire $[t(19)=$ $8.48, p<.001$ and $t(19)=3.74, p<.01$, respectively]. There was no significant difference between the performance test and the questionnaire for the simple tasks in either the rule-finding instruction or the standard instruction group. Pearson correlations were also calculated by combining the rule-finding instruction subjects
Table 4

Results for Experiment 2: Mean Correct Percentages on the Performance Test $(P)$ and the Questionnaire $(Q)$, and Mean Trials to Targets $(T ; N=80)$

\begin{tabular}{llll}
\hline \multicolumn{1}{c}{ Instructions } & $P$ & $Q$ & $T$ \\
\hline Standard & Complex Task & & \\
Rule finding & .60 & .30 & 9.46 \\
& .60 & .13 & 7.47 \\
& Simple Task & & \\
Standard & .29 & .42 & 8.47 \\
Rule finding & .58 & .68 & 6.67 \\
\hline
\end{tabular}

and the standard instruction subjects. The correlation between the performance test and questionnaire was highly significant in the simple task $(r=.76, p<.001)$ and was not significant in the complex task. This experiment replicated the findings of Experiment $1 \mathrm{~A}$ that subjects showed implicit learning in the complex task and that no implicit learning was involved in the simple task. Subjects in the simple rule-number condition were fully aware of what they had learned.

As for the effect of instructions, for the complex tasks, the rule-finding group did worse than the standard group on the questionnaire $[t(38)=2.4, p<.05]$. No significant difference between these two groups of subjects was found on the performance test. On the other hand, for the simple task, the rule-finding group did better than the standard group on both the performance test and the questionnaire $[t(38)=3.21, p<.01$ and $t(38)=$ $1.97, p<.05$, respectively]. In addition, the postexperiment written reports by subjects showed that none of the subjects in the complex task wrote down the correct pattern (i.e., the equations relating the input numbers and output numbers). On the other hand, for the simple task, the number of subjects ( 10 out 20 ) who found the correct rule in the rule-finding group was significantly greater than the number in the standard group [ 3 out of 20 ; $\left.\chi^{2}(1)=4.10, p<.05\right]$.

For the simple task, instructing subjects to search for the patterns between input values and output values significantly improved their performance test and the ability to answer the questionnaire. The postexperiment reports revealed parallel results. These results suggest that rule-finding instructions increased motivation and encouraged subjects to use a conscious strategy, which enhanced explicit learning. For the complex task, a negative effect was found on the questionnaire, and no effect was found on the performance test. This was consistent with Reber's (1976) suggestion that it was almost impossible for subjects to figure out the correct rules, due to the complexity of the stimulus structures. Explicitly searching for the patterns produced a strong tendency for subjects to infer or invent rules that were not accurate representations of stimuli. In other words, this kind of instruction caused subjects to mislead themselves and further impaired their performance on the task. 


\section{EXPERIMENT 3}

The second learning context adopted by the present study consisted of asking subjects to verbalize their thinking processes during the training. Berry and Broadbent (1984) showed that verbalization alone did not improve subjects' task performance or question answering. However, both Stanley et al. (1989) and McGeorge and Burton (1989) found that verbalization could have a significant positive effect on task performance. Different amounts of practice and contextual effects created by the surface structures of the tasks among these studies might explain the contradictory results. Yet it remains unclear how verbalization influences implicit and explicit learning. The goal of Experiment 3 was to address this issue.

Three levels of verbalization have been suggested (Ericsson \& Simon, 1984). A first level of verbalization is simply the vocalization of covert articulatory or oral encodings. A second level involves description, or explication, of the thought content. Most studies using the first and the second levels of verbalization show that verbalization had no effect on performance or on the structure of the thought process (see review by Ericsson \& Simon). A third level of verbalization requires subjects to explain their thought processes or thoughts. This level signif icantly improves performance on the transfer task in the Tower of Hanoi problem, in which disks of different sizes are moved among three pegs (Gagne \& Smith, 1962; Wilder \& Harvey, 1971). It therefore seems that by inducing more deliberate planning and forcing subjects to think, the third level of verbalization would change the nature and course of the thinking process. Moreover, it was predicted that this kind of change would improve explicit learning, in the same way that the learning of the Tower of Hanoi problem does. On the other hand, this kind of change might limit subjects to the explicit mode of thinking and further impair implicit learning.

\section{Method}

Subjects and Design. See Experiment $1 \mathrm{~A}$ for details regarding subjects. Eighty-eight subjects participated in this experiment. There were two between-subjects factors: task type (complex vs. simple) and verbalization type (silent vs. verbalization). Testing type (performance test vs. questionnaire) was a within-subjects factor. The subjects were randomly assigned to one of the following groups: a silent group for the complex task, a silent group for the simple task, a verbalization group for the complex task, and a verbalization group for the simple task

Procedure. All the procedures were the same as in Experiment 2, except for the following two differences. First, the results in Experiment 2 showed that for the simple task, very few subjects in the standard group had found the rules. To avoid a floor effect, all subjects in Experiment 3 were told that there were patterns that determined the relationships between the inputs and the outputs. However, they were neither encouraged to find the patterns nor informed about the testing of the patterns. The emphasis was still on finding the correct inputs for each pair of target outputs. This was the only difference between the silent group of the present experiment and the standard instruction group of Experiment 2.

Second, during the learning phase for the verbalization groups, after subjects finished the first question, they were asked to think aloud while solving the remaining 11 questions. They were not asked to think aloud on the first question, to give them an opportunity to become familiar with the task before they started to verbalize. The following extra instructions were given to subjects in the verbalization group:

I want you to tell me in detail about how you do all the problems. Try to think aloud. You probably often do this when you are alone and working on a problem. I am particularly interested in the reasons that you select your answers, so try to give me the reasons for each response that you make, any kind of reason. Do not be afraid to say if you are guessing. I will tape record all of what you say.

The subjects were encouraged to talk. If they stopped for more than $30 \mathrm{sec}$, the experimenter would ask them to state the reasons that they made their choice. The purpose of the tape recording was to promote talking

\section{Results and Discussion}

The results are presented in Table 5. First, there was no significant difference on the mean trials to targets among different conditions. Second, a $2 \times 2 \times 2$ ANOVA showed that there was a significant difference on the correct percentages between the performance test and the questionnaire $\left[F(1,84)=17.80, M S_{\mathrm{e}}=.05, p<\right.$ $.001]$, while there were no reliable effects involving verbalization type. The interaction between task type (complex vs. simple) and testing type (performance test vs. questionnaire) was also highly significant $[F(1,84)=$ $\left.28.01, M S_{\mathrm{e}}=.05, p<.001\right]$. There was no three-way interaction.

Finally, planned comparisons showed that for the complex task, subjects in both the verbalization group and the silent group did significantly better on the performance test than on the questionnaire $[t(21)=3.10$, $p<.01$ and $t(21)=5.96, p<.001$, respectively]. There was no significant difference between the performance test and the questionnaire for the simple tasks in either the verbalization group or the silent group. When the verbalization group and the silent group were combined, the Pearson correlation between the performance test and the questionnaire was highly significant in the simple task $(r=.78, p<.001)$ and not significant in the complex task. Thus, both implicit learning from the complex rule-line task and explicit learning from the simple rule-number task were once again demonstrated in Experiment 3.

With regard to the effect of verbalization, for the simple tasks, the verbalization group did better than the silent group on the performance test $[t(42)=1.95, p<$

Table 5

Results for Experiment 3: Mean Correct Percentages on the Performance Test $(P)$ and the Questionnaire $(Q)$, and Mean Trials to Targets $(T ; N=88)$

\begin{tabular}{llll}
\hline Group & $P$ & $Q$ & $T$ \\
\hline & Complex Task & & \\
Silent & .55 & .25 & 6.58 \\
Verbalization & .44 & .28 & 5.73 \\
& Simple Task & & \\
Silent & .38 & .46 & 7.96 \\
Verbalization & .59 & .60 & 6.39 \\
\hline
\end{tabular}


$.05]$ and on the questionnaire, but without reaching statistical significance in the latter. For the complex task, there was no difference between the verbalization group and the silent group on either measure.

These results indicate that verbalization facilitates explicit learning and has no effect on implicit learning. This supports the idea that the explicit process-forcing subjects to think and reason verbally-improves explicit learning. In the complex-task condition, the tape-recording protocol showed that for most of the responses, the reason that subjects gave for their choice was "just guessing." Asking subjects to verbalize seems not to cause much effect on subjects' learning. Accordingly, no difference was found between the verbalization and silent groups.

If subjects' knowledge learned from the complex task was conscious, they should be able to answer the questionnaire as they did in the simple task, regardless of the way in which they represented this knowledge. There is nothing to suggest that verbalization would be more difficult, even if the representation were visual and relational, because the questionnaire tested only quantitative relationships among four variables.

The present experiment provides additional support for the assertion that the complex task and the simple task involve two different learning systems. In the complex task, subjects are not aware of either how they learn or what they have learned, and they have difficulty in putting into words both how they learn and what they have learned. It seems that when the stimulus structures are complex and the coding format encourages perceptual discrimination, the learning becomes a more passive and automatic process, and it is difficult for subjects to make that learning process explicit. On the other hand, subjects are consciously aware of their learning process when the relationships between variables are simple and the learning involves symbolic manipulation.

\section{EXPERIMENT 4}

The goal of Experiment 4 was to investigate the effect of observational learning on both implicit and explicit learning. According to the findings of Reber and his colleagues (e.g., Reber \& Lewis, 1977), implicit learning could happen in several different situations. Subjects improved their grammaticality judgment after processing the letter strings by either memorization (Reber, 1967, 1976; Reber \& Lewis, 1977), associate learning, or observation (Reber \& Allen, 1978) during the learning phase. It seems that at least in some cases, implicit learning takes place in a natural and passive way. Implicit learning might occur whenever subjects devote sufficient attention to the stimulus, without employing conscious decision-making and hypothesis-testing strategies. On the other hand, active decision-making and hypothesis-testing processes would be critical for explicit learning.

The present experiment employed an observationallearning procedure, in which subjects learned the rela- tionship between inputs and outputs in a passive way without involving a decision-making process, to examine whether learning still occurs for the complex and simple tasks. More importantly, if learning occurs, how does this observational-learning procedure change subjects' performance on both learning tasks?

\section{Method}

Subjects and Design. Ninety-six subjects participated in this experiment. They were seniors at Chin-Ou High School in Taipei, Taiwan.

Two between-subjects factors were task type (complex vs. simple) and learning-context condition (observational learning vs. standard). Testing type (performance test vs. questionnaire) was a within-subjects factor. The subjects were randomly assigned to one of the following four groups: two standard groups-one for the complex task and one for the simple task; and two observational learning groups- - one for the complex task and one for the simple task.

Procedure. The procedure for the standard group in the present experiment was the same as that used for the silent group of Experiment 3 . The pairs of input and output numbers produced by the standard group for each trial on all 12 questions were given to the observational-learning subjects.

The observational-learning groups in both the complex and simple tasks also employed the same procedures, with the following difference: In each trial, for all the 12 questions, in addition to the target values, the subjects were also presented with the value for Input 1 and asked to type in the same number. The same procedure was repeated for Input 2 . The numbers for Input 1 and Input 2 were given by the yoked control subjects-the standard group. The observational-learning subjects had no choice concerning what values they were going to enter, and only had to type whatever numbers appeared on the screen. The purpose of asking subjects to type numbers was to ensure that they paid attention to the numbers and to make the observational-learning groups more comparable to the standard groups. The following change of instructions was added for these subjects: "Your task is to observe the numbers of input and output shown on the computer screen and to enter the same numbers." The subjects were also informed that there would be tests after the learning section and were encouraged to watch closely the changes in the output values. This was in order to further ensure that they paid attention to both input and output values.

\section{Results and Discussion}

The results are presented in Table 6 . On average, it took both the observational-learning and the yoked control subjects 9.37 trials $(S D=2.20)$ for the complex task, and 6.20 trials $(S D=1.90)$ for the simple task to reach the targets in each question. As to the mean correct percentages, a $2 \times 2 \times 2$ ANOVA showed a main effect of learning condition [standard vs. observational learning; $\left.F(1,92)=10.00, M S_{\mathrm{e}}=.20, p<.01\right]$ and an interaction effect between type of learning task (complex vs. simple) and testing type [performance test vs. questionnaire; $\left.F(1,92)=13.60, M S_{\mathrm{e}}=0.06, p<.001\right]$.

The planned comparisons revealed that for the complex task, the standard group did significantly better on the performance test than on the questionnaire $[t(23)=$ $4.96, p<.001]$, and that no such difference was found in the observational-learning conditions. These results indicate that implicit learning occurred only in the standard condition, and not in the observational-learning condition. 
Table 6

Results for Experiment 4: Mean Correct Percentages on the Performance Test $(P)$ and the Questionnaire $(Q)$, and Mean Trials to Targets $(T ; N=96)$

\begin{tabular}{lccc}
\hline \multicolumn{1}{c}{ Group } & $P$ & $Q$ & $T$ \\
\hline & Complex Task & & \\
Standard & .57 & .30 & 9.37 \\
Observational learning & .33 & .22 & 9.37 \\
& Simple Task & & \\
Standard & .37 & .45 & 6.20 \\
Observational learning & .14 & .19 & 6.20 \\
\hline
\end{tabular}

As to the simple task, for neither the standard group nor the observational-learning group were there significant differences between the performance test and the questionnaire. These results suggest that no implicit learning occurred. The subjects' performance could be fully accounted for by their explicit knowledge. However, for the observational-learning group, the mean correct percentages on both the questionnaire and the performance test were not significantly above chance. Accordingly, only the standard group showed explicit learning, and virtually no learning occurred for the observational-learning subjects.

As for the effect of learning condition, for the complex task, the standard group did significantly better than the observational learning group on the performance test $[t(46)=4.69, p<.001]$. No such difference was found on the questionnaire. For the simple task, on the other hand, there were significant differences between the standard group and the observational-learning group on both the questionnaire $[t(46)=2.75, p<.01]$ and the performance test $[t(46)=2.06, p<.05]$. The standard subjects did significantly better than the observational-learning subjects on both measures.

These results indicate that in the simple rule-number condition, active participation in decision-making processes was necessary for learning to occur. In the complex rule-line condition, learning still occurred without decision-making processes, even though it was impaired. More importantly, subjects' explicit knowledge could account for their performance test results, indicating that no implicit learning occurred.

The results from the observational learning in the complex task were inconsistent with the original hypotheses. This may be attributed to the differences between the implicit-learning paradigms. Both the acquisition processes and acquired knowledge in the task used in the present study were different from those in the task used in Reber's paradigm (e.g., Reber, 1967; Reber \& Allen, 1978). It seems that the knowledge produced by Reber's paradigm is similar to concept learning (Mathews et al., 1988; Reber \& Allen, 1978). In this case, implicit learning could take place in a natural and passive way. The control-task paradigm used in the present study produced a mental model (Sanderson, 1989; Stanley et al., 1989). Subjects learned something about the relationship between inputs and outputs with practice. It is pos- sible that active decision-making processes became crucial for implicit learning to occur in these kinds of tasks. This assertion is consistent with the findings of Berry (1991), who also used a control-task paradigm.

It is difficult to study observational learning, because even though subjects are instructed to watch all the variables closely, it is hard to control what subjects actually do while they are observing. In the present experiment, however, the observational-learning subjects for the complex task did significantly better than chance on both the performance test and the questionnaire, and their scores on the questionnaire did not significantly differ from those of the standard group. It seems unlikely that these results would have been obtained if subjects had not paid attention to the outputs. Still, to ensure that the problem of inattentiveness can be ruled out, further improvements in the procedure would be needed.

\section{GENERAL DISCUSSION}

\section{The Implicit-Explicit Distinction of Learning}

The first main findings of the present study seem to suggest that the complex task and simple task involve different types of learning. In the complex task, the measure of subjects' task performance was always better than the measure of their explicit knowledge, indicating that implicit learning occurred. In the simple task, the subjects' task performance was either equal to or worse than their explicit knowledge, suggesting that no implicit learning was involved and that sometimes the subjects did not use their explicit knowledge completely or efficiently. The dissociation between the performance test and the questionnaire in the complex-task condition, and the association between these two measures in the simple-task condition, were further evidenced by the correlational data found in all the experiments.

Implicit learning, which occurred in the complex task, is procedural and perceptual in nature. Subjects iterated their way to a perfect match of line lengths with targets. Their explicit knowledge could not fully account for their performance. Thus, at least part of their acquired knowledge was unavailable to their conscious awareness. On the other hand, explicit learning, which occurred in the simple task, involves the manipulation of symbols and results in the conscious representation of underlying rules. However, since explicit knowledge was tested after the learning phase during which subjects completed the questionnaire, the present study cannot rule out the possibility that subjects' knowledge is not learned explicitly, but instead is made explicit at the time of the test. This would be more likely to happen in the simple task than in the complex task, which would provide an alternative explanation of the above interpretation.

The distinction between tasks and processes needs to be pointed out here. The present findings indicate that implicit learning occurred in the complex task and not in the simple task. However, subjects did acquire some explicit knowledge in the complex task. Thus, it seems that both implicit and explicit processes operate in perform- 
ing the complex task used in the present study. The acquisition processes and resulting knowledge for any given tasks may involve both modes of learning (Broadbent et al., 1986). In other words, all learning lies somewhere along the implicit-explicit continuum. The question is, what factors are crucial in determining the point on this continuum? Implicit learning has been demonstrated under various conditions (e.g., Berry \& Broadbent, 1984; Lewicki, 1982; Reber, 1989b). Reber (1989a) and Broadbent et al. (1986) suggest that the complexity of the stimulus is the important factor. The coding format of the stimulus has been neglected by most researchers. The tasks used in the present study provide a way in which to investigate both complexity and coding format of the stimulus. The results suggest that both are necessary to determine the type of learning involved.

The procedure used to demonstrate implicit learning in the present study is similar to that used in one of the experiments in Berry and Broadbent (1987). Both lines of research measure performance and explicit knowledge regarding the same aspect of a task. In Berry and Broadbent (1987), during the learning phase, subjects had to enter input to get a target value of output. Berry and Broadbent demonstrated implicit learning by showing that subjects did better on a transfer task, in which they were also required to enter input to get a target value of output, than they did on answering questions about the relationship between input and output on questionnaires. The present study includes several improvements that provide better control for demonstrating implicit learning.

First, instead of testing the performance and administering a questionnaire in two different groups of subjects and conditions, the present study compared these two measures within the same subjects and conditions. This provides more convincing evidence for the existence of implicit learning. Implicit learning is demonstrated when there is a dissociation between subjects' performance and explicit knowledge. However, if the conditions in which subjects' performance and explicit knowledge are assessed are incompatible, dissociation will not be meaningful. In this case, dissociation could be caused by the incompatibility of testing conditions rather than by subjects' lack of explicit knowledge about tasks. For example, in both Berry and Broadbent (1987) and the present studies, subjects learned the task by interacting with a computer. If only the performance had been tested on the computer, with the questionnaire having been administered in another fashion, it is possible that higher scores on the performance test than on the questionnaire would have been due simply to the fact that performance was tested in a condition that was more similar to the learning condition.

Another improvement of this study was that neither measure in the present study was influenced by subjects' prior knowledge regarding the relationship between variables. In Berry and Broadbent (1987), the questionnaire indicated that subjects had had some prior knowledge about the relationship between variables. It is pos- sible that higher scores on the performance test than on the questionnaire were due to prior knowledge rather than to knowledge gained as a result of task experience. This problem was avoided in the present study by using meaningless labels ( $x$ and $y$ ).

One reason for testing both the complex and simple tasks was to verify the validity of the paradigm used in the present study. The learning and testing procedures of the complex and simple tasks were virtually identical. The only differences were the complexity of rules and the coding format. In the simple task, subjects did as well on the questionnaire as they did on the performance test. This suggests that the questionnaire was sufficient to assess subjects' explicit knowledge used in the performance test. A more direct verification of the validity of the explicit measure-when subjects were given the equations in the complex task-showed once again that the questionnaire was sensitive enough to assess their explicit knowledge. The finding that subjects sometimes did not use their explicit knowledge completely or efficiently suggests that the explicit measure may be even more sensitive than the performance test. Thus, the finding that the performance test was better than the explicit measure cannot be attributed to the performance test being more sensitive than the questionnaire.

\section{Effects of Learning Contexts}

The second main set of findings of the present study was that verbalization and instructions to search for the rule, both of which promote an explicit process, facilitate the simple-task learning and hurt or have no effect on the complex-task learning. For the observationallearning condition, which induced an implicit mode of learning, no learning occurred in the simple task, and learning was impaired and became explicit in the complex task. Thus, the explicit-learning contexts have opposite effects on the complex and simple tasks. The observational-learning context, on the other hand, has parallel effects on the complex and simple tasks.

These results have several important implications. First, explicit processes have a differential effect on the complex and simple tasks. This is consistent with the assertion that complex and simple tasks involve two distinct learning systems. The second implication concerns the role of metacognitive processes, which are executive processes used in planning, monitoring, and decision making in task performance (Flavell, 1979). The function of the explicit processes, which lead to more deliberate and systematic decision making and hypothesis testing, is to encourage metacognitive activities. Metacognitive knowledge, which allows subjects to use strategies more flexibly and to be more aware of their learning processes, enhances the learning in the simple rule-number condition. On the other hand, the acquisition process of the complex rule-line condition is implicit. Metacognitive activities do not play an important role, and sometimes even impair learning.

Finally, both implicit learning and explicit learning require active participation of their learning processes. 
With only action and no decision-making or hypothesistesting processes, no learning occurred in the simple task, and learning was hurt in the complex task. This is consistent with Berry (1991) and also with the argument that the incidental (passive)-intentional distinction of learning is not parallel to the implicit-explicit learning distinction. In the current case, subjects' intention is required for implicit learning to occur.

In the standard simple task, what subjects did was very much like the processes of hypotheses testing. They could keep testing their hypotheses by entering different numbers and looking at the results. In the observationallearning condition, however, it seems much more difficult for subjects to keep track of the information required to form reasonable hypotheses. An even more interesting finding was that no implicit learning occurred in the complex rule-line condition. Subjects' performance was as good as their explicit knowledge. Without involving decision-making processes, subjects may have more cognitive resources available while observing. This would have allowed them to become more aware of the relationship between input and output variables. Thus, there was no implicit learning. This explanation is based on ideas that implicit learning occurs when there is a large amount of information that needs to be considered (Broadbent et al., 1986) and that the amount of information that causes implicit learning is relative to subjects' processing capacity available during learning. The first idea has been supported by many studies on implicit learning (e.g., Reber, 1989a). The second idea, however, needs to be tested further.

\section{Conclusion}

The present study used questionnaires to measure explicit knowledge. This procedure reduces both the problem of suggesting answers to subjects, as yes-no recognition does, and the problem that subjects may not have sufficient verbal skill to report their explicit knowledge. Compared with previous studies, the present study also provides better controls and more well-defined conditions to demonstrate implicit learning and explicit learning.

The complex task and simple task seem to involve two kinds of learning. Verbalization and instructions to search for rules have different effects on these two tasks, and both tasks require subjects' active participation in the learning processes.

\section{REFERENCES}

Alten, R., \& Reber, A. S. (1980). Very long term memory for tacit knowledge. Cognition, 8, 175-195.

BERRY, D. C. (1991). The role of action in implicit learning. Quarterly Journal of Experimental Psychology, 43A, 881-906.

Berry, D. C., \& Broadbent, D. E. (1984). On the relationship between task performance and verbal knowledge. Quarterly. Journal of Experimental Psychology, 36A, 209-231

Berry, D. C., \& Broadbent, D. E. (1987). The combination of implicit and explicit learning processes. Psychological Research, 49, 7-15.

Berky, D. C., \& Broadrent, D. E. (1988). Interactive tasks and the implicit-explicit distinction. British Journal of Psychology, 79, 251272.

BroadBent, D. E. (1977). Levels, hierarchies, and the locus of control. Quarterly Journal of Experimental Psychology, 29A, 181-201.

Broadbent, D. E., \& AsTon, B. (1978). Human control of a simulated economic system. Ergonomics, 21, 1035-1043.

Broadbent, D. E., FitzGeral.i, P., \& Broadbent, M. H. P. (1986). Implicit and explicit knowledge in the control of complex systems. British Journal of Psychology, 77, 33-50.

Brody, N. (1989). Unconscious learning of rules: Comment on Reber's analysis of implicit learning. Journal of Experimental Psychology: General, 118, 236-238.

Carlson, R. A., \& Dulany, D. E. (1985). Conscious attention and abstraction in concept learning. Journal of Experimental Psychology: Learning. Memory, \& Cognition, 11, 45-58.

Dulany, D. E., Carlson, R. A., \& Dewey, G. I. (1984). A case of syntactical learning and judgment: How conscious and how abstract? Journal of Experimental Psychology: General, 113, 541-555.

Dulany, D. E., Carlson, R. A., \& Dewey, G. I. (1985). On consciousness in syntactical learning and judgment: A reply to Reber, Allen, and Regan. Journal of Experimental Psychology: General, 114, 25-32.

Ericsson, K. A., \& Simon, H. A. (1984). Protocol analysis: Verbal reports as data. Cambridge, MA: MIT Press.

Flavell, J. (1979). Metacognition and cognitive monitoring. American Psychologist, 34, 906-911.

Gagne, R. M., \& Smith, E. C. (1962). A study of the effects of verbalization on problem solving. Journal of Experimental Psychology, 63, $12-18$.

LEWICKI, P. (1982). Trait relationships: The nonconscious generalization of social experience. Personality \& Social Psychology Bulletin, 8, 439-445.

LEWICKI, P. (1986a). Nonconscious social information processing. New York: Academic Press.

LEWICKI, P. (1986b). Processing information about covariations that cannot be articulated. Journal of Experimental Psychology: Learning, Memory, \& Cognition, 12, 135-146.

Lewicki, P., Czyzewska, M., \& Hoffman, H. (1987). Unconscious acquisition of complex procedural knowledge. Journal of Experimental Psychology: Learning. Memory, \& Cognition, 13, 523-530.

Lewicki, P., Hill, T., \& Bizot, E. (1988). Acquisition of procedural knowledge about a pattern of stimuli that cannot be articulated. Cognitive Psychology, 20, 24-37.

Mathews, R. C. (1990). Abstractness of implicit grammar knowledge: Comments on Perruchet and Pacteau's analysis of synthetic grammar learning. Journal of Experimental Psychology: General, 118, 412-416.

Mathews, R. C., Buss, R. R., Chinn, R., \& Stanley, W. B. (1988). The role of explicit and implicit learning processes in concept discovery. Quarterly Journal of Experimental Psychology, 40A, 135165.

Mathews, R. C., Buss, R. R., Stanley, W. B., Blanchard-Fields, F., Cho, J. R., \& Druhan, B. (1989). Role of implicit and explicit processes in learning from examples: A synergistic effect. Journal of Experimental Psychology: Learning, Memory, \& Cognition, 15, 1083-1100.

MCGeorge, P., \& Burton, A. M. (1989). The effects of concurrent verbalization on performance in a dynamic systems task. British Journal of Psychology, 80, 455-465.

Millward, R. B., \& Reber, A. S. (1972). Probability learning: Contingent-event sequences with lags. American Journal of Psychologi, 85, 81-98.

Perruchet, P., \& Amorim, M.-A. (1992). Conscious knowledge and changes in performance in sequence learning: Evidence against dissociation. Journal of Experimental Psychology: Learning, Memory, \& Cognition, 18, 785-800.

Perruchet, P. \& Pacteau, C. (1990). Synthetic grammar learning: Implicit rule abstraction or explicit fragmentary knowledge? Journal of Experimental Psychology: General, 119,264-275.

REBER. A. S. (1967). Implicit learning of artificial grammars. Joumal of Verbal Learning \& Verbal Behavior, 77, 317-327.

REBER, A. S. (1976). Implicit learning of synthetic languages: The role 
of instructional set. Journal of Experimental Psychology: Human Learning \& Memory, 2, 88-94.

REBER, A. S. (1989a). Implicit learning and tacit knowledge. Journal of Experimental Psychology: General, 118, 219-235.

REBER, A. S. (1989b). More thoughts on the unconscious: Reply to Brody and to Lewicki and Hill. Journal of Experimental Psychology: General, 118, 242-244.

Reber, A. S., \& Allen, R. (1978). Analogy and abstraction strategies in synthetic grammar learning: A functionalist interpretation. Cognition, 6, 189-221.

Reber, A. S., Allen, R., \& Regan, S. (1985). Syntactical learning and judgment, still unconscious and still abstract: Comment on Dulany, Carlson, and Dewey. Journal of Experimental Psychology: General, 114, 17-24.

Reber, A. S., \& Lewis, S. (1977). Toward a theory of implicit learning: The analysis of the form and structure of a body of tacit knowledge. Cognition, 5, 333-361.

REBER, A. S., \& MILLWARD, R. B. (1971). Event of tracking in probability learning. American Journal of Psychology, 84, 85-99.

SAnderson, P. M. (1989). Verbalizable knowledge and skilled task performance: Association, dissociation, and mental models. Journal of Experimental Psychology: Learning, Memory, \& Cognition, 4, 729-747.

SCHACTER, D. L. (1987). Implicit memory: History and current status. Journal of Experimental Psychology: Learning, Memory, \& Cognition, 13, 501-518.

SQUire, L. R., \& Frambach, M. (1990). Cognitive skill learning in amnesia. Psychobiology, 18, 109-117.

STADLER, M. (1989). On learning complex procedural knowledge. Journal of Experimental Psychology: Learning, Memory, \& Cognition, 15, 1061-1069.

Stanley, W. B., Mathews, R. C., Buss, R. R., \& Kotler-Cope, S. (1989). Insight without awareness: On the interaction of verbalization, instruction and practice in a simulated process control task. Quarterly Journal of Experimental Psychology, 41A, 553-577.

WILDER, L., \& HARVEY, D. J. (1971). Overt and covert verbalization in problem solving. Speech Monographs, 38, 113-120.

(Manuscript received July 12, 1994; accepted for publication September 16, 1994.) 